\title{
Lohnt sich betriebliche Suchtprävention?
}

\section{Zu Effektivität und Effizienz betrieblicher Alkoholprävention}

\author{
Is Prevention of Substance Abuse for Employees Cost-Beneficial? - Effectiveness

\section{and Efficiency of Alcohol Prevention for Employees}

\author{
Simone Rey-Riek ${ }^{1}$ \\ Franziska Güttinger ${ }^{1}$ \\ Jürgen Rehm ${ }^{1,2,3}$
}

\section{Zusammenfassung}

Zielsetzung: Mitarbeitende mit Suchtproblemen können den betroffenen Betrieben erhebliche Kosten verursachen. Um erfolgreich zu sein, müssen sich präventive Maßnahmen jedoch für Betriebe in betriebswirtschaftlicher Hinsicht „lohnen“. Methode: Mittels einer umfassenden Literaturrecherche wurden Arbeiten aus dem angelsächsischen und hauptsächlich dem deutschen Sprachraum zu Effektivität und Effizienz von betrieblichen Maßnahmen im Alkoholbereich begutachtet. Ergebnisse: Es zeigte sich, dass nur wenige Arbeiten aus dem deutschsprachigen Raum vorliegen. Durch alkoholbedingte Fehlzeiten verursachte Kosten stehen im Mittelpunkt der meisten Studien. Auch wenn die Studien untereinander schlecht vergleichbar sind, wird aufgezeigt, dass durch präventive Maßnahmen ein wirtschaftlicher Nutzen entsteht, der die Kosten solcher Maßnahmen übersteigt. Schlussfolgerungen: Im Sinne eines eher primärpräventiven Ansatzes, der die Betriebe relativ wenig kostet und sich als effektiv erwiesen hat, wird für die Schweiz die Einführung von Kurzinterventionen bei Personen mit risikoreichem Alkoholkonsum auch auf betrieblicher Ebene vorgeschlagen.

\section{Schllïsselwörter}

Suchtprävention · Betriebe · Alkohol · Effizienz · Effektivität

\section{Abstract}

Objective: As employees with addiction problems may cause substantial costs to companies, cost-effective measures are needed in order to prevent these costs. The overall objective of this study was to evaluate the cost-benefit ratio of preventive measures in the workplace with potential application for Switzerland. Methods: A computer-assisted comprehensive literature search and review were carried out on publications which dealt with topics on effectiveness, cost-effectiveness and cost-benefit studies in Anglo-Saxon and German-speaking countries. We also looked at grey literature published in German. Results: There is a scarcity of studies from German-speaking countries. In addition, the majority of the studies on alcohol-attributable costs at the workplace were restricted to absenteeism and did not include all costs of alcohol. Even though studies were not easily comparable, there seems to be an economic benefit from preventive measures, outweighing their costs. Conclusions: A measure between primary and secondary prevention which requires relatively few resources and has proven to be effective are brief interventions. It is recommended that companies in Switzerland introduce this measure as a way of dealing with alcohol problems among their employees.

\section{Key words}

Prevention of substance abuse - workplace - alcohol - efficiency · effectiveness

${ }^{1}$ Institut für Suchtforschung (ISF), Zürich, Schweiz

${ }^{2}$ Centre for Addiction and Mental Health, Toronto, Kanada

${ }^{3}$ Public Health Sciences, University of Toronto, Kanada

Korrespondenzadresse

Simone Rey-Riek · Institut für Suchtforschung · Konradstraße 32/Postfach · 8031 Zürich, Schweiz ·

E-mail: msrey@dplanet.ch 
Betriebe sind wichtige potenzielle Akteure im alkoholpräventiven Handeln, denn der Arbeitsplatz ist einer der relevanten Lebensbereiche, die mit alkoholbedingten Beeinträchtigungen konfrontiert sind. Obwohl die Gesundheit der Mitarbeitenden im praktischen Alltag oft kein zentraler Punkt in der Arbeitswelt ist, gibt es gute Gründe, weshalb Gesundheitsförderung und Prävention im Betrieb Beachtung geschenkt werden soll. Zum einen wirkt sich die Arbeit in erheblichem Maße auf das Wohlbefinden und die Gesundheit der Menschen aus, und zwar sowohl in positiver als auch in negativer Hinsicht; zum anderen bietet der Arbeitsplatz einen guten Zugang, um gezielte gesundheitsfördernde bzw. präventive Maßnahmen umzusetzen. Unabhängig davon, ob im Bereich der Primär-, Sekundär- oder Tertiärprävention Maßnahmen ergriffen werden, sollte die Entwicklung von Präventionsprogrammen in jedem Fall auf empirisch gewonnenen Forschungserkenntnissen basieren (science-based or evidencebased prevention; siehe [1]).

In betriebswirtschaftlicher Hinsicht können Mitarbeitende mit Suchtproblemen erhebliche direkte als auch indirekte Kosten verursachen. Dabei fallen unter anderem Kosten für Kurzfehlzeiten und krankheitsbedingte Ausfälle, Personalersatz während solcher Ausfälle, quantitative und qualitative Minderleistung, Schädigung Dritter durch alkoholbedingte Fehlhandlungen usw. an [2]. In der heutigen wirtschaftlichen Situation ist zudem der Legitimationsdruck bei betrieblichen Maßnahmen zur Suchtbekämpfung stärker geworden. Deshalb sind Wirksamkeit (Effektivität) und Wirtschaftlichkeit (Effizienz) der Programme wichtige Kriterien, wenn es um die Einführung von Präventionsprogrammen geht [3].

Aus dieser Situation heraus stellt sich die zentrale Frage: Lohnt sich Suchtprävention für Betriebe? Gibt es betriebliche Suchtpräventionsprogramme, die erwiesenermaßen kostensparend und wirksam sind? Im Rahmen einer Überblicksarbeit soll die verfügbare empirische Evidenz zusammengetragen und die Übertragbarkeit besonders Erfolg versprechender Maßnahmen auf die besonderen Verhältnisse in der Schweizer Arbeitswelt diskutiert werden. Bisherige Erfahrungen in der betrieblichen Suchtprävention und Ergebnisse in Bezug auf Effizienz und Effektivität von Alkoholpräventionsprogrammen sollen als Grundlage für Empfehlungen für das künftige praktische Vorgehen dienen. Die Empfehlungen für schweizerische Betriebe könnten in einem weiteren Schritt auch für andere Länder diskutiert werden.

\section{Methodik}

Geplant war eine systematische Metaanalyse deutschsprachiger Literatur zur Effektivität und Effizienz von betrieblichen Maßnahmen im Alkoholbereich.

Die Literaturrecherche zur Identifikation von praxisnahen Studien zur Alkoholprävention in Betrieben erfolgte mittels Datenbanken (Medline, ETOH, SOMED, Nebis), durch Anschrift von internationalen ExpertInnen und durch Suche auf Homepages von Institutionen, die im Gesundheitsbereich tätig sind. Die Literaturrecherche betraf die Jahre 1965 bis heute.
Eingeschlossen wurden empirische Arbeiten qualitativer und quantitativer Art, theoretische Abhandlungen sowie Bibliografien und Monografien. Die einbezogenen Studien mussten in Form eines veröffentlichten Artikels oder schriftlichen Berichtes vorliegen. Teilweise wurde auf Beschreibungen in Sekundärliteratur zurückgegriffen, da ein Teil der Kosten-Nutzen-Analysen nicht in Originalform zugänglich war.

Die ursprünglich geplante Studie erwies sich als nicht durchführbar, da zu wenige Artikel aus dem deutschsprachigen Raum vorliegen, die eine systematische Analyse ermöglicht hätten. Zudem war ein systematischer Vergleich der einzelnen Studien fast unmöglich, da große Unterschiede in den betrieblichen Hintergründen, Interventionen und Untersuchungsdesigns bestanden. Anstelle einer quantitativen Metaanalyse erfolgte deshalb eine qualitative Zusammenfassung der Forschungsergebnisse unter Berücksichtigung nordamerikanischer Literatur und eine allgemeine Einbettung dieser Ergebnisse in die Thematik der betrieblichen Suchtprävention. Dabei ist vorauszuschicken, dass sich die meisten berücksichtigten Präventionsprogramme auf Alkohol beziehen, doch nicht in allen Fällen ausschließlich auf diese Substanz beschränkt sind.

\section{Betriebliche Suchtprävention}

Die vorhandene Literatur zum Thema „betriebliche Suchtprävention“ zeigt, dass in den letzten zwei Jahrzehnten in Nordamerika die so genannten EAP (Employee Assistance Programs) am meisten Bedeutung erlangt haben $[4,5]$. Unter dem Namen EAP verbirgt sich eine Anzahl unterschiedlicher Programme, die nicht nur auf Alkohol- oder andere Suchtprobleme, sondern auch auf weitere häufige psychosoziale Problemfaktoren ausgerichtet sind. Den EAP liegt kein allgemein gültiges Konzept zugrunde; vielmehr unterscheiden sie sich in der Art und Weise ihres Schwerpunktes oder im Aufwand, der betrieben wird, um auffällig gewordene MitarbeiterInnen zu motivieren und zu reintegrieren. Ein wichtiges Element der prototypischen EAP ist die so genannte „konstruktive Konfrontation“ [4, 5]. Das heißt, der oder die Vorgesetzte thematisiert gegenüber der untergebenen Person das arbeitsbezogene Fehlverhalten bzw. die Abnahme der Leistungsfähigkeit. Diese Konfrontation soll außerdem dazu führen, ein Hilfsangebot zu vermitteln. Es handelt sich also hier bereits um ein sekundärpräventives Instrument, das klassischerweise nur eingesetzt wird, wenn bereits Probleme oder Symptome vorhanden sind. Neben der Intervention durch den Arbeitgeber oder Vertreter des Managements ist es aber ebenso wichtig, dass diese Programme Personen ermutigen, sich selbst zu melden, anstelle „gezwungen“ zu werden.

Das Konzept des „konstruktiven Druckes“ wurde in Deutschland aus nord-amerikanischen Ansätzen übernommen und bisher erfolgreich umgesetzt. In der Schweiz sind Suchtpräventionsprogramme dagegen meist Teil von allgemeinen Gesundheitsförderungsprogrammen. Konkrete Hilfsangebote für Mitarbeitende mit Suchtproblemen, die gewisse Qualitätsstandards erfüllen, sind eher die Ausnahme [6]. 


\section{Studien aus Nordamerika}

Im Gegensatz zu anderen Ländern wurde im nordamerikanischen Raum eine größere Anzahl von Kosten-Nutzen-Analysen von Programmen bzw. Interventionen durchgeführt. Trotzdem gibt es auch für Nordamerika bisher keine zusammenfassende und vollständige Wirkungsforschung von EAP-Programmen [4, 7].

Zur Effektivität und Kosteneffizienz von EAP und Alkoholpräventionsprogrammen in den USA liegen zwei Übersichtsarbeiten vor, nämlich von Kurtz et al. [8] und Colantonio [9]. Kurtz et al. erarbeiteten eine Übersicht zum Erfolg von Alkoholpräventionsprogrammen in Betrieben. Sie analysierten elf Studien aus der Zeitperiode von Ende der 50er-Jahre bis 1984. Colantonio berücksichtigte in ihrer Untersuchung zur Effektivität von EAP dreizehn seit 1975 publizierte Studien. Beide Autoren kommen zum Schluss, dass zwar die einbezogenen Studien von deutlichen Kosteneinsparungen durch betriebsbezogene Betreuungsprogramme ausgehen; nach Meinung von Kurtz und seinen Kollegen ist es jedoch schwierig zu beweisen, dass betriebliche Alkoholprogramme tatsächlich die Kosten reduzieren, was sie vor allem mit den mangelhaften Studiendesigns der berücksichtigten Untersuchungen begründen.

Auch andere Autoren [10 - 13] kommen zu dem Schluss, dass ein Zusammenhang zwischen der Anwendung einer konstruktiven Konfrontationsstrategie und verbesserter Arbeitsleistung besteht und dass EAP-Programme alkoholabhängige und -missbrauchende MitarbeiterInnen einer Behandlung zuführen können.

\section{Studien aus Deutschland}

In der Bundesrepublik Deutschland werden betriebliche Suchtpräventionsprogramme in der Regel nicht evaluiert, weshalb lediglich einzelne exemplarische Untersuchungen oder Beschreibungen vor allem aus Großbetrieben vorliegen. Wie bereits einleitend angemerkt, sind die Studien schwer vergleichbar, da sie auf unterschiedlichen Studiendesigns beruhen. Die vorliegenden Analysen konzentrieren sich zudem hauptsächlich auf die durch Fehlzeiten verursachten Kosten (einen Überblick über diverse Studien geben Petschler und Fuchs [14]) und ziehen damit andere alkoholbedingte Folgen nicht in Betracht. Selbst bei solch eingeengter Sichtweise zeigen Kosten-Nutzen-Analysen jedoch, dass die alkoholbedingten Fehlzeiten in einem Maße reduziert werden konnten, dass wirtschaftlicher Nutzen durch Interventionsmaßnahmen erreicht werden konnte. Studien mit detaillierteren Wirtschaftlichkeitsberechnungen, die auch andere Kostenfaktoren einbeziehen, liegen jedoch für Deutschland nicht vor.

\section{Studien aus der Schweiz}

Wie die durchgeführte Literaturrecherche ergab, werden auch in der Schweiz betriebliche Suchtpräventionsprogramme in der Regel nicht evaluiert. Eine gute Zusammenfassung von Schweizer Studien zur betriebswirtschaftlichen Relevanz von betrieblichen Suchtpräventionsprogrammen findet sich ebenfalls in einer $\mathrm{Ar}$ beit von Fuchs und Petschler [2].
Eine der wenigen schweizerischen Arbeiten, die für betriebliche Suchtprävention relevant sind, ist bereits etwas älter und stammt von Mühlemann [15]. Dieser vergleicht in einer umfangreichen und sorgfältig aufgebauten Fall-Kontroll-Studie eine Gruppe AlkoholikerInnen ( $\mathrm{n}=64)$ mit einer dreimal so großen Kontrollgruppe ( $n=192$ ) hinsichtlich betrieblicher Fehlzeiten und Krankheit. Einbezogen wurde die Belegschaft aus zwei Schweizer Bundesbetrieben. Datengrundlage bildeten Informationen aus der umfangreichen Kartei des Bundesärztlichen Dienstes, in der neben Zahl und Dauer der Fehlzeiten, Gründen und Diagnosen der damals rund 117000 Bundesangestellten alle medizinisch relevanten Dokumente erfasst waren. Bei den 64 als AlkoholikerInnen gekennzeichneten Personen war zwischen 1967 und 1975 die ärztliche Diagnose „chronische Alkoholabhängigkeit“ festgehalten worden. Der Kontrollgruppe gehörten 192 MitarbeiterInnen an, die in Bezug auf den Arbeitsplatz nie im Zusammenhang mit Alkohol auffällig gewesen waren und die in mehreren Eigenschaften wie z. B. Dienststelle, Alter, Sprache, mit den AlkoholikerInnen übereinstimmen mussten.

Für den 10-jährigen Überprüfungszeitraum ergaben sich deutlich höhere Fehltage für die AlkoholikerInnen. Die Fallgruppe wies 2,6-mal mehr Abwesenheitstage infolge Krankheit oder Unfall und 1,9-mal mehr Abwesenheitstage infolge Kurzfehlzeiten (bis 3 Tage) auf als die Kontrollgruppe, wobei die Kurzfehlzeiten nur ein Fünftel der Gesamtfehlzeiten bei AlkoholikerInnen ausmachten. Zusammengenommen fehlte ein Alkoholiker durchschnittlich 30 Tage, eine Kontrollperson knapp 12 Tage pro Jahr. Der rein „alkoholbedingte“ Mehraufwand für den Betrieb betrug in dieser Studie 2288,- sFr (ca. 1525,- Euro) pro Person und Jahr.

\section{Kostenrechnung}

Polli [16] berechnete die alkoholbedingten Kosten für einen Betrieb mit einer Belegschaft von 100 MitarbeiterInnen basierend auf verschiedenen Einzelergebnissen aus den USA, der Bundesrepublik Deutschland und der Schweiz. Er ging von 4,3\% Alkoholabhängigen in der Gesamtbelegschaft aus und nahm für Alkoholabhängige eine pauschale Leistungsmindestseinbuße von einem Drittel an. In seiner Berechnung kommt Polli zu einer Gesamtbelastung für den Betrieb von 235000,- sFr (ca. 157 000,- Euro) pro Jahr bzw. 2350,- sFr pro Mitarbeiter/Jahr. Unter Hinzurechnung eines Ersatzkostenanteils (für frühzeitiges Ausscheiden) von 860,- sFr (ca. 570,- Euro) geht Polli von einem jährlichen Gesamtschaden für den Betrieb pro Mitarbeiter/Jahr von 3210,- sFr (ca. 2140,- Euro) aus. Über einen Zeitraum von 10 Jahren belaufen sich die Kosten für ein von ihm vorgeschlagenes Betreuungsprogramm auf 29500,- sFr (ca. 19 667,- Euro) pro MitarbeiterIn gegenüber einem zu erwartenden Gesamtschaden von $32100,-\mathrm{sFr}$ (ca. 21 400,- Euro) pro Mitarbeiter bei „Nichthandeln“ des Betriebes. Die „Nutzschwelle“ wird nach seinem Berechnungsmodell nach 4,3 Jahren erreicht.

Als grobe Faustregel zur Berechnung der betrieblichen Gesamtkosten durch alkoholbedingte Fehlzeiten, Arbeitsunfähigkeit sowie Arbeitsunfälle wird häufig das vom Stanford Research Institute (SRI) entwickelte Modell herangezogen. Dieses macht die durch Alkoholprobleme verursachte Kosten von der Stellung des 
Mitarbeiters/der Mitarbeiterin abhängig und geht von einem globalen Minderungsfaktor von 25\% des Gehaltes aus [2].

Sowohl Fuchs und Petschler [2] als auch Wienemann [17] weisen darauf hin, dass auf der Basis dieser Formel die Höhe der Schätzung gegenüber den realen Kosten eher als zu niedrig anzusehen ist, da darin die weniger leicht quantifizierbaren Kosten wie etwa niedrigere Arbeitsqualität, Betriebsstörungen und Stillstände, Fehlentscheidungen, schlechtes Betriebsklima oder Ersatz für arbeitsunfähige und gekündigte Beschäftigte gar nicht erfasst worden seien. Somit kommt diese Formel nur einer groben Schätzung gleich, da deren empirische Basis als viel zu schmal und für verallgemeinerbare Aussagen als ungenau angesehen werden muss.

\section{Diskussion}

Ziel dieser Überblicksarbeit war die Frage nach der Effizienz und Effektivität betrieblicher Suchtprävention und es interessierte vor allem, was bisherige Erfahrungen und Ergebnisse für die Übertragung auf schweizerische Betriebe beitragen können.

Die breite Literatursuche in verschiedenen Datenbanken zeigte, dass insbesondere im europäischen Raum ein Mangel an Studien zu Wirtschaftlichkeit und Wirksamkeit betrieblicher Präventionsmaßnahmen im Suchtbereich herrscht. Nordamerikanische, deutsche und schweizerische Studien, die hauptsächlich auf der Fehlzeitenproblematik basieren, ergeben, dass sich Prävention betriebswirtschaftlich lohnt. Will man jedoch den ökonomischen Nutzen von betrieblichen Suchtpräventionsprogrammen umfassender beschreiben, so stößt man mit den üblichen ökonomischen Methoden an Grenzen, weil eine ganze Reihe von Größen (z.B. Minderleistung, Schädigung Dritter etc.) eine Rolle spielt, die nicht sehr einfach in Geldwerten zu fassen sind. Man braucht deshalb erweiterte Wirtschaftlichkeitsberechnungen, die versuchen, eine erheblich größere Bandbreite von Faktoren einzubeziehen, was allerdings aufwändige Erfassungs- und Analyseverfahren voraussetzt.

EAP (Employee Assistance Programs) haben sich gemäß den Analysen insbesondere in Großbetrieben mit über 1000 Beschäftigten bewährt. In der Schweiz sind rund zwei Drittel der ArbeitnehmerInnen in kleinen und mittleren Unternehmen (gemäß EU-Definition Unternehmen mit weniger als 250 Beschäftigten) tätig [18]. Es besteht deshalb die Notwendigkeit, spezielle Angebote für kleine und mittelgroße Unternehmen zu entwickeln.

Wie sich gezeigt hat, setzen EAP-Programme bei der Erkennung von Alkoholproblemen relativ spät ein. Es ist jedoch bekannt, dass eine erkennbare Abnahme der Arbeitsleistung sowie eine Häufung der Fehlzeiten ein vergleichsweise spätes Anzeichen von Alkoholproblemen bei Mitarbeitenden ist. Roman und seine Kollegen [7] weisen ebenfalls darauf hin, dass sich die meisten Programme eher dem Erkennen von Alkoholproblemen und deren Behandlung (d.h. der Sekundärprävention) als der Vorbeugung (d.h. der Primärprävention) widmen. Hier müssten geeignete Wege der Intervention gefunden werden, um alkoholgefährdete Personen in einer frühen Phase des Verlaufes zu erkennen und zu erreichen.
Vorschlag für die Schweiz

Im Folgenden soll für die Schweiz ein Präventionsmodell vorgeschlagen werden, das die oben genannten Probleme bei der Umsetzung von Maßnahmen in schweizerischen Betrieben berücksichtigt und sich daher besser für die spezifische Situation in der Schweiz eignen könnte: die so genannten Kurzinterventionen oder „brief interventions“. Dieses Modell geht nicht von einer Behandlung von bereits erkrankten, d.h. abhängigen oder missbrauchenden Personen aus, sondern von Interventionen, die sich explizit an stark Trinkende oder Problemtrinker wenden. Unter die Zielgruppe fallen üblicherweise Frauen, die im Durchschnitt mehr als $20 \mathrm{~g}$ reinen Alkohols pro Tag zu sich nehmen, und Männer, die mehr als $40 \mathrm{~g}$ zu sich nehmen [19, 20]. Mit anderen Worten: Es handelt sich um eine Intervention, die wesentlich breiter und früher als die bisher beschriebenen Interventionen angelegt ist.

Solche Kurzinterventionen werden üblicherweise von ÄrztInnen oder anderen Fachleuten in Gesundheitsberufen durchgeführt und dienen der Früherkennung und Beratung von Personen mit risikoreichem Alkoholkonsum [21]. Dabei werden PatientInnen auf ihren risikoreichen Alkoholkonsum aufmerksam gemacht und Hilfe sowie weiterführende Informationen werden angeboten [22].

In einer breit angelegten WHO-Studie wurde gezeigt, dass Kurzinterventionen in viele verschiedene Kulturen übertragbar sind [23], und mehrere Metaanalysen haben bisher ihre Effektivität nachgewiesen [21, 24-26]. Neuere Untersuchungen bestätigen die Nachhaltigkeit dieser Effekte [27] und die generelle Kosteneffektivität dieser Interventionsform [27, 28].

Kurzinterventionen werden in der Schweiz bereits angewendet und finden im Rahmen der Alkoholprävention Eingang in Fortbildungsprogramme für Ärzte der medizinischen Grundversorgung [29] (vgl. auch die Publikationen des Teilprojektes Ärzte der nationalen Alkoholpräventionskampagne „Alles im Griff“: www.alles-im-griff.ch). Als besonders geeignet für die Prävention sind Kurzinterventionen, weil sie zusätzlich zur Reduktion von Durchschnittskonsum sich auch dazu eignen, „Binge“-Konsum, d.h. Trinkgelegenheiten mit hohem Einmalkonsum, zu vermindern (z. B. [27]). Binge-Konsum ist neben hohem Durchschnittskonsum für einen beträchtlichen Teil der alkoholbedingten Gesundheitsschäden verantwortlich, insbesondere im Bereich der Unfälle (z. B. [20]; siehe auch [30, 31]).

Bezüglich der Wirksamkeit von Kurzinterventionen wurden in einer WHO-Studie [23] Effekte von 10\% und mehr - je nach Ergebniskriterium - gefunden; in der Metaanalyse von Babor et al. [21] wurde beispielsweise ein mehr als doppelt so großer Effekt gefunden. Die Effekte zeigten sich bereits bei Kurzinterventionen von fünf Minuten Dauer.

In der Schweiz verursachten Problemtrinker 553,6 Mio. sFr (ca. 369,1 Mio. Euro) direkte Krankheitskosten und 92,4 Mio. sFr (ca. 61,6 Mio. Euro) direkte Verkehrsunfallkosten (jeweils konservative Schätzungen für 1998, siehe [32]). Daraus ergibt sich pro Problemtrinker pro Jahr nach der Definition von English et al. [19] mehr als 2000,- sFr (ca. 1334,- Euro) an direkten Kosten 
(Grundlagen siehe [33], für die Aufteilung der AlkoholkonsumentInnen in die Trinkkategorien siehe [19]; UN-Zahlen für Einwohner in der Schweiz). Bei einer Reduktion um 10\% ergibt sich ein Nutzen von 200,- sFr pro Trinker (ca. 133,- Euro). Eine fünfminütige Intervention kostet inklusive Vorbereitung deutlich weniger. Mit anderen Worten: Nur schon aufgrund der direkten Krankheitskosten lohnt sich eine Intervention für Problemtrinker - auch im Betrieb.

Die genannten Zahlen sind sehr konservative Schätzungen. Zum einen wurden nur direkte Gesundheitskosten mit einbezogen. Wie oben ausgeführt, entstehen aber im Betrieb durch Alkohol ganz andere Kosten wie Produktionsausfälle durch die Fehltage, Unfallfolgen und anderes. Zum Zweiten wurden nur die Kosten für ein Jahr mit einbezogen. Wie Fleming et al. [27] zeigen, sind Kurzinterventionen aber durchaus über ein Jahr hinaus wirksam. Und schließlich wurde hinsichtlich der Effektivität von Kurzinterventionen der niedrigste Wert aus der Literatur herangezogen. Absichtlich wurden also immer konservative Annahmen gewählt, um zu zeigen, dass selbst unter diesen Annahmen der Nutzen einer solchen Intervention die Kosten immer noch übersteigt.

Insgesamt kann sich betriebliche Prävention also lohnen, vor allem dann, wenn sie sich auf empirisch gestützte Konzepte wie Kurzinterventionen stützen kann. Die vorgeschlagene Strategie ist auch deshalb von Wert, weil sie unabhängig vom finanziellen Gewinn früh interveniert und so manifester Alkoholabhängigkeit und Alkoholmissbrauch, wie in ICD definiert, präventiv begegnen kann. Wir schlagen deshalb eine empirische Untersuchung in der Schweiz vor, die die beschriebene Kurzintervention in Betrieben einführt und hinsichtlich Effektivität und Kosten-Nutzen-Verhältnis aus Sicht der Betriebe evaluiert.

\section{Danksagung}

Diese Studie entstand mit finanzieller Unterstützung des Instituts für Sozial- und Präventivmedizin der Universität Zürich, Abteilung Prävention und Gesundheitsförderung, sowie der Suchtpräventionsstelle der Stadt Zürich. Wir danken diesen beiden Stellen herzlich für ihre Unterstützung und die gute Zusammenarbeit.

\section{Literatur}

${ }^{1}$ Brounstein PJ, Zweig JM, Gardner SE. Science-based Substance Abuse Prevention. A Guide. http://www.dmhas.state.ct.us/sig/pdf/pubs_ Guide.pdf (15.10.2002)

${ }^{2}$ Fuchs R, Petschler T. Betriebswirtschaftliche Kosten durch Alkoholmissbrauch und Alkoholabhängigkeit. In: Fuchs R, Rainer L, Rummel M (Hrsg). Betriebliche Suchtprävention. Göttingen: Verlag für Angewandte Psychologie 1998; 51 - 76

${ }^{3}$ Fuchs R, Rainer L, Rummel M et al. Betriebliche Suchtprävention: Ein Arbeitsfeld in der Diskussion. In: Fuchs R, Rainer L, Rummel M (Hrsg). Betriebliche Suchtprävention. Göttingen: Verlag für Angewandte Psychologie 1998; $13-29$

${ }^{4}$ Roman PM, Blum TC. Dealing with alcohol problems in the workplace. Recent Developments in Alcoholism 1993; 11: 473-491

${ }^{5}$ Klepsch R, Fuchs R. Entwicklung und Entwicklungsstand amerikanischer und kanadischer Alkohol-Präventionsprogramme. In: Fuchs R, Rainer L, Rummel M (Hrsg). Betriebliche Suchtprävention. Göttingen: Verlag für Angewandte Psychologie 1998; 257-279
${ }^{6}$ Buchmann H, Müller R. Alkohol- und Tabakprävention in Schweizer Unternehmen. Bericht über eine repräsentative Betriebsbefragung in der Schweiz. Lausanne: SFA-ISPA, Schweizerische Fachstelle für Alkohol- und andere Drogenprobleme 2001

${ }^{7}$ Roman PM, Blum TC. The workplace and alcohol problem prevention. Alcohol Research \& Health 2002; 26 (1): 49-57

${ }^{8}$ Kurtz NR, Googins BG, Howard WC. Measuring the success of occupational alcoholism programs. Journal of Studies of Alcohol 1984; 45 (1): $33-45$

${ }^{9}$ Colantonio A. Assessing the effects of employee assistance programs: a review of employee assistance programs evaluations. The Yale Journal of Biology and Medicine 1989; 62 (1): $13-22$

${ }^{10}$ Finley D. Anxiety and the alcoholic. Social Work 1972; 17: 29-34

${ }^{11}$ Heymann M. Referral to alcoholism programs in industry: coercion, confrontation and choice. Journal of Studies on Alcohol 1976; 37: 900-908

${ }^{12}$ Heymann M. Alcoholism Programs in Industry. New Brunswick: N.J.L. Publications Division, Rutgers Center for Alcohol Studie 1978

${ }^{13}$ Freedberg EJ, Johnston WE. Changes in drinking behavior, employment status and other life areas for employed alcoholics three, six and twelve months after treatment. Journal of Drug Issues 1979; 9: $523-534$

${ }^{14}$ Petschler T, Fuchs R. Betriebswirtschaftliche Kosten- und Nutzenaspekte innerbetrieblicher Alkoholprobleme. Sucht aktuell 2000; 1 (7): $14-18$

${ }^{15}$ Mühlemann R. Betriebliche Prävention von Alkoholismus in schweizerischen Bundesbetrieben. In: Deutsche Hauptstelle gegen die Suchtgefahren (Hrsg). Suchterkrankung am Arbeitsplatz. Früherkennung und Behandlung. Hamm: Hoheneck-Verlag 1983; 176- 186

${ }^{16}$ Polli E. Betriebliche Sekundärprophylaxe. Kreuzlingen: VSFA, Schweiz. Verband von Fachleuten für Alkoholgefährdeten- und Suchtkrankenhilfe 1988

17 Wienemann E. Suchtprävention und -hilfe im Betrieb - ein profitabler Kostenfaktor? In: Brosch R, Mader R (Hrsg). Alkohol am Arbeitsplatz. Wien: Orac 2000

${ }^{18}$ Bundesamt für Wirtschaft und Arbeit seco. www.kmuinfo.ch/ index.html? Art $=$ SEITE1.1\&SeiteID = 03.02_struktur_nach_groesse (2.9.2002)

${ }^{19}$ English DR, Holman CDJ, Milne E et al. The quantification of drug caused morbidity and mortality in Australia 1995. Canberra, Australia: Commonwealth Department of Human Services and Health 1995

${ }^{20} \mathrm{Rehm}$ J, Room R, Monteiro M et al. Alcohol as a risk factor for burden of disease. In: WHO (Hrsg). Comparative quantification of health risks: global and regional burden of disease due to selected major risk factors. Geneva: WHO (im Druck)

${ }^{21}$ Babor T, Caetano R, Casswell S et al. Alcohol: no ordinary commodity a consumer's guide to public policy. Oxford: Oxford University Press (im Druck)

22 WHO Department of Mental Health and Substance Dependence. Screening and brief intervention for alcohol problems in primary care. World Health Organization 2001

${ }^{23}$ WHO Brief Interventions Study Group. A cross-national trial of brief interventions with heavy drinker. Am J Public Health 1996; 86: 949-955

24 Wilk AI, Jensen NM, Havighurst TC. Meta-analysis of randomised control trials addressing brief interventions in heavy alcohol drinkers. J Gen Intern Med 1997; 12: 274-283

25 Poikolainen K. Effectiveness of brief interventions to reduce alcohol intake in primary health care populations: a meta-analysis. Prev Med 1999; 28: $503-509$

${ }^{26}$ Moyer A, Finney JW, Swearingen CE et al. Brief interventions for alcohol problems: a meta-analytic review of controlled investigations in treatment-seeking and non treatment-seeking populations. Addictions 2002; 97: 279-292

${ }^{27}$ Fleming MF, Mundt MP, French MT et al. Brief physician advice for problem drinkers: long-term efficacy and benefit-cost analysis. Alcoholism: Clinical and Experimental Research. 2002; 26: 36-43

28 Fleming MF, Mundt MP, French MT et al. Benefit-cost analysis of brief physician advice with problem drinkers in primary care settings. Medical Care 2000; 38: 7-18

${ }^{29}$ Stoll B, Daeppen , JB et al. Die Kurzintervention: eine gezielte Beratung von Patienten mit übermäßigem Alkoholkonsum. Praxis 1999; 88: $1699-1704$ 
${ }^{30}$ Cherpitel CJ, Tam T, Midanik L et al. Alcohol and nonfatal injury in the U.S. general population: a risk function analysis. Accident Analysis Prevention 1995; 27: 651-661

${ }^{31}$ Cherpitel CJ. Drinking pattern and problems and drinking in the event: an analysis of injury by cause among casualty patients. Alcoholism: Clinical and Experimental Research 1996; 20: 1130-1137
${ }^{32}$ Jeanrenaud C, Priez F, Pellegrini S et al. Le coût social de l'abus d'alcool en Suisse. Neuchâtel: Université de Neuchâtel 2001

${ }^{33}$ Gutjahr E, Gmel G. Die sozialen Kosten des Alkoholkonsums in der Schweiz. Epidemiologische Grundlagen 1995-1998. Lausanne: Schweizerische Fachstelle für Alkohol- und andere Drogenprobleme SFA/ISPA 2001 